\title{
On the Computation of Correctly-Rounded Sums
}

\author{
P. Kornerup \\ V. Lefèvre \\ N. Louvet \\ J.-M. Muller \\ $S D U$, Odense, Denmark \\ LIP, CNRS/ENS Lyon/INRIA/UCBL/Université de Lyon, Lyon, France
}

\begin{abstract}
This paper presents a study of some basic blocks needed in the design of floating-point summation algorithms. In particular, in radix-2 floating-point arithmetic, we show that among the set of the algorithms with no comparisons performing only floatingpoint additions/subtractions, the 2Sum algorithm introduced by Knuth is minimal, both in terms of number of operations and depth of the dependency graph. We investigate the possible use of another algorithm, Dekker's Fast2Sum algorithm, in radix-10 arithmetic. We give methods for computing, in radix 10 , the floating-point number nearest the average value of two floating-point numbers. We also prove that under reasonable conditions, an algorithm performing only round-to-nearest additions/subtractions cannot compute the round-to-nearest sum of at least three floating-point numbers. Starting from an algorithm due to Boldo and Melquiond, we also present new results about the computation of the correctly-rounded sum of three floating-point numbers. For a few of our algorithms, we assume new operations defined by the recent IEEE 754-2008 Standard are available.
\end{abstract}

Keywords: Floating-point arithmetic, summation algorithms, correct rounding, 2Sum and Fast2Sum algorithms.

\section{INTRODUCTION}

The computation of sums appears in many domains of numerical analysis. Examples are numerical integration, evaluation of dot products, matrix products, means, variances and many other functions. When computing the sum of $n$ floating-point numbers $a_{1}, a_{2}, \ldots, a_{n}$, the best one can hope is to get $\circ\left(a_{1}+a_{2}+\cdots a_{n}\right)$, where $\circ$ is the desired rounding function (specified by a rounding mode, or by a rounding direction attribute, in the terminology of the IEEE 754 Standard for floating-point arithmetic [2], [11]). On current architectures this can always be done in software using multiple-precision arithmetic. This could also be done using a long accumulator, as advocated by Kulisch [4], but such accumulators are not available on current processors.

It is well known that the rounding error generated by a round-to-nearest addition is itself a floating-point number. Many summation algorithms published in the literature (see for instance [1], [18], [19], [17], [5], [22]) are based on this property and implicitly or explicitly use basic blocks such as Dekker's Fast2Sum and Knuth's 2Sum algorithms (Algorithms 1 and 2 below) to compute the rounding error generated by a floating-point addition. Since efficiency is one of the main concerns in the design of floating-point programs, we focus on algorithms using only floating-point additions and subtractions in the target format and without conditional branches, because on current pipelined architectures, a wrong branch prediction may cause the instruction pipeline to drain, with a resulting drastic performance loss. The computation of the correctly-rounded sum of three floating-point numbers is also a basic task needed in different contexts: in [5], Boldo and Melquiond presented a new algorithm for this task, with an application in the context of the computation of elementary functions. Hence, it is of great interest to study the properties of these basic blocks.

In this paper, we assume an IEEE 754 [2], [11] arithmetic. We show that among the set of the algorithms with no comparisons performing only floating-point operations, the 2Sum algorithm introduced by Knuth is minimal, both in terms of number of operations and depth of the dependency graph.

The recent revision of the IEEE Standard for floatingpoint arithmetic considers arithmetics of radices 2 and 10 . Some straightforward properties of radix- 2 arithmetic have been known for a long time and are taken for granted. And yet, some properties do not hold in radix 10. A simple example is that, in radix 10 , computing the average value of two floating-point numbers $a$ and $b$ first by computing $a+b$ rounded to the nearest, and then by computing half the obtained result rounded to the nearest again will not necessarily give the average value rounded to the nearest. We will investigate that problem and suggest some strategies for accurately computing the average value of two numbers in decimal arithmetic.

Under reasonable assumptions, we also show that it is impossible to always obtain the correctly round-to-nearest sum of $n \geq 3$ floating-point numbers with an algorithm performing only round-to-nearest additions/subtractions. The algorithm proposed by Boldo and Melquiond for computing the round-to-nearest sum of three floating-point numbers relies on a non-standard rounding mode, rounding to odd (see Definition 1). We show that if the radix is even, rounding to odd can be emulated in software using only floating-point additions/subtractions in the standard rounding modes and a multiplication by the constant 0.5 , thus allowing the round-to-nearest sum of three floatingpoint numbers to be determined without tests. We also propose algorithms to compute the correctly-rounded sum of three floating-point values for directed roundings.

In a preliminary version of this paper [12], we gave results valid in radix-2 floating-point arithmetic. We now extend these results to other radices (the most interesting one being radix 10), consider the problem of computing an 
average value in radix 10, give new summation algorithms, and extend the results of Theorems 2 and 3 to any precision.

\section{A. Assumptions and notations}

We assume a radix- $\beta$ and precision- $p$ floating-point arithmetic as defined (for radices 2 and 10) in the IEEE 754-2008 standard [11]. Typical examples are the basic formats defined by that standard: precisions 24, 53 or 113 in radix 2, and 7,16 and 34 in radix 10 . The user can choose an active rounding mode, also called rounding direction attribute: round toward $-\infty$, round toward $+\infty$, round toward 0, round to nearest "even", which is the default rounding mode, and round to nearest "TiesToAway" 1 . Given a real number $x$, we denote respectively by $R D(x), R U(x), R Z(x)$ and $R N(x)$ the rounding functions associated to these rounding direction attributes (assuming round to nearest even for $R N(x)$ ).

Correct rounding is required for the four elementary arithmetic operations and the square root by the above cited IEEE standards: an arithmetic operation is said to be correctly rounded if for any inputs its result is the infinitely precise result rounded according to the active rounding mode. Correct rounding makes arithmetic deterministic, provided all computations are done in the same format, which might be sometimes difficult to ensure [15]. Correct rounding allows us to design portable floating-point algorithms and to prove their correctness, as the results summarized in the next subsection.

Given a real number $x \neq 0, \operatorname{ulp}_{p}(x)$ denotes the unit in the last place of $x$, i.e., if $\beta^{e} \leq|x|<\beta^{e+1}$ with $e \in \mathbb{Z}$, then $\operatorname{ulp}_{p}(x)=\beta^{e+1-p}$. Where there is no ambiguity on the value of $p$, we just write $\operatorname{ulp}(x)$.

We assume in the following that no double roundings occur (that is, that all computations take place in the same working precision). For instance, users of GNU/Linux on 32 -bit x 86 processors should be aware that by default, all the computations on their platform are carried out in the so-called double-extended precision (64 bits) format. ${ }^{2}$ However, such processors tend to be less and less common, and the x86-64 architecture does not have such problems in practice, due to the use of the SSE instructions by default for single and double precision arithmetic. These issues are discussed with details in Chapter 7 of [16], and the recent IEEE 754-2008 Standard requires that it should be possible, if desired, to perform all intermediate computations in a given format.

\footnotetext{
${ }^{1}$ A tie-breaking rule must be chosen when the real number $x$ to be rounded falls exactly halfway between two consecutive floating-point numbers. A frequently chosen tie-breaking rule is round to nearest even: $x$ is rounded to the only one of these two consecutive floatingpoint numbers whose significand is even. This is the default mode in the IEEE 754-2008 Standard. The standard also defines another tie-breaking rule, required in radix 10, called round ties to away: $x$ is rounded to the one of the two consecutive numbers whose significand has the largest magnitude

${ }^{2}$ Depending on the context, intermediate results may or may not be converted to the target format, but both behaviors are here regarded as incorrect.
}

\section{B. Previous results}

The Fast2Sum algorithm (Algorithm 1) was introduced by Dekker [8] in 1971, but the three operations of this algorithm already appeared in 1965 as a part of a summation algorithm, called "Compensated sum method," due to Kahan [1]. The following result is due to Dekker [8], [16].

Theorem 1 (Fast2Sum algorithm): Assume a radix- $\beta$ floating-point arithmetic, with $\beta \leq 3$, with subnormal ${ }^{3}$ numbers available, that provides correct rounding with rounding to nearest. Let $a$ and $b$ be finite floating-point numbers, both nonzero, such that the exponent of $a$ is larger than or equal to that of $b$. If $a+b$ does not overflow, then the following algorithm computes floatingpoint numbers $s$ and $t$ such that $s=R N(a+b)$ and $s+t=a+b$ exactly.

$$
\begin{aligned}
\text { Algorithm } 1 \text { (Fast2Sum }(a, b)) \text { : } & \begin{aligned}
s & =R N(a+b) ; \\
z & =R N(s-a) ; \\
t & =R N(b-z) ;
\end{aligned}
\end{aligned}
$$

Note that underflow cannot hinder the result: this is a rather straightforward consequence of a fact that if $x$ and $y$ are radix- $\beta$ floating-point numbers, and if the number $R N(x+y)$ is subnormal, then $R N(x+y)=x+y$ exactly (see [9] for a proof in radix 2, which easily generalizes to higher radices). Also, the only overflow that may occur is when adding $a$ and $b$.

Note that instead of having information on the exponents, one may know that $|a| \geq|b|$, but in such a case, the condition of the theorem is fulfilled. Also, the condition $\beta \leq 3$ restricts the use of this algorithm in practice to binary arithmetic. That condition is necessary: consider, in radix 10, with precision $p=4$, the case $a=b=9999$. By applying Fast2Sum, we would get $s=20000$ and $t=-1$. This gives $s+t=19999$, whereas $a+b=19998$.

However, if a wider internal format is available (one more digit of precision is enough), and if the computation of $z$ is carried on using that wider format, then the condition $\beta \leq 3$ is no longer necessary. This might be useful in decimal arithmetic, when the target format is not the largest one that is available in hardware. We discuss the possible use of Fast2Sum in radix 10 in Section III.

If no information on the relative orders of magnitude of $a$ and $b$ is available, or if the radix is larger than 3, there is an alternative algorithm due to Knuth [13] and Møller [14], called 2Sum.

$$
\begin{aligned}
\text { Algorithm 2 (2Sum } & (a, b)) \text { : } \\
s & =R N(a+b) ; \\
b^{\prime} & =R N(s-a) ; \\
a^{\prime} & =R N\left(s-b^{\prime}\right) ; \\
\delta_{b} & =R N\left(b-b^{\prime}\right) ; \\
\delta_{a} & =R N\left(a-a^{\prime}\right) ; \\
t & =R N\left(\delta_{a}+\delta_{b}\right) ;
\end{aligned}
$$

\footnotetext{
${ }^{3}$ In an arithmetic of precision $p$, a subnormal number has the form $M \cdot \beta^{e_{\min }-p+1}$, where $e_{\min }$ is the smallest possible exponent and $M$ is an integer such that $0<|M| \leq \beta^{p-1}-1$, as opposed to a normalized number of the form $M \cdot \beta^{e-p+1}$, where $e_{\min } \leq e \leq e_{\max }$ and $M$ is an integer such that $\beta^{p-1} \leq|M|<\beta^{p}$.
} 
2Sum requires 6 operations instead of 3 for the Fast2Sum algorithm, but on current pipelined architectures, a wrong branch prediction may cause the instruction pipeline to drain. As a consequence, using 2Sum instead of a comparison followed by Fast2Sum will usually result in much faster programs [17]. The names "2Sum" and "Fast2Sum" seem to have been coined by Shewchuk [21]. They are a particular case of what Rump [20] calls "errorfree transforms". We call these algorithms error-free additions in the sequel.

The IEEE 754-2008 standard [11] describes some new operations with two floating-point numbers as operands:

- minNum and maxNum, which deliver respectively the minimum and the maximum;

- minNumMag, which delivers the one with the smaller magnitude (the minimum in case of equal magnitudes);

- maxNumMag, which delivers the one with the larger magnitude (the maximum in case of equal magnitudes).

The operations minNumMag and maxNumMag can be used to sort two floating-point numbers by order of magnitude, without using comparisons or conditional branches. In radices less than or equal to three (or when a wider precision is available for computing $z$ ), this leads to the following alternative to the 2Sum algorithm.

Algorithm $3(\operatorname{Mag} 2 \operatorname{Sum}(a, b))$ :

$$
\begin{aligned}
s & =R N(a+b) \\
a^{\prime} & =\operatorname{maxNumMag}(a, b) ; \\
b^{\prime} & =\operatorname{minNumMag}(a, b) ; \\
z & =R N\left(s-a^{\prime}\right) \\
t & =R N\left(b^{\prime}-z\right) ;
\end{aligned}
$$

Algorithm Mag2Sum consists in sorting the inputs by magnitude before applying Fast2Sum. It requires 5 floating-point operations, but the first three operations can be executed in parallel. Mag2Sum can already be implemented efficiently on the Itanium processor, thanks to the instructions famin and famax available on this architecture [7, p. 291]. Notice that, since it is based on the Fast2Sum algorithm, Algorithm Mag2Sum does not work in radices higher than 3. Also, when underflow and overflow are concerned, it has the same properties as Fast2Sum: underflow is harmless, and the only overflow that may occur is when computing $R N(a+b)$.

\section{Algorithms 2Sum And Mag2Sum ARE Minimal IN RADIX 2}

In the following, we call an $R N$-addition algorithm an algorithm only based on additions and subtractions in the round-to-nearest mode: at step $i$ the algorithm computes $x_{i}=R N\left(x_{j} \pm x_{k}\right)$, where $x_{j}$ and $x_{k}$ are either one of the input values or a previously computed value. An RNaddition algorithm must not perform any comparison or conditional branch, but may be enhanced with minNum, maxNum, minNumMag or maxNumMag as in Theorem 3.
For instance, 2Sum is an RN-addition algorithm that requires 6 floating-point operations. To estimate the performance of an algorithm, only counting the operations is a rough estimate. On modern architectures, pipelined arithmetic operators and the availability of several FPUs make it possible to perform some operations in parallel, provided they are independent. Hence the depth of the dependency graph of the instructions of the algorithm is an important criterion. In the case of Algorithm 2Sum, only two operations can be performed in parallel, $\delta_{b}=$ $R N\left(b-b^{\prime}\right)$ and $\delta_{a}=R N\left(a-a^{\prime}\right)$. Hence the depth of Algorithm 2Sum is 5. In Algorithm Mag2Sum the first three operations can be executed in parallel, hence this algorithm has depth 3 .

In this section we address the following question: are there other RN-addition algorithms producing the same results as 2Sum, i.e., computing both $R N(a+b)$ and the rounding error $a+b-R N(a+b)$ for any floating-point inputs $a$ and $b$, that do not require more operations, or that have a dependence graph of smaller depth?

We have shown the following result, proving that among the RN-addition algorithms, 2Sum is minimal in terms of number of operations as well as in terms of depth of the dependency graph.

Theorem 2: Consider a binary arithmetic in precision $p \geq 2$. Among the RN-addition algorithms computing the same results $s$ and $t$ as 2Sum on any inputs,

1) each one requires at least 6 operations;

2) each one with 6 operations reduces to 2 Sum through straightforward transformations (symmetries, etc.);

3) each one has depth at least 5 .

As previously mentioned an RN-addition algorithm can also be enhanced with minNum, maxNum, minNumMag and maxNumMag operations [11], which is the case for Algorithm Mag2Sum. The following result states the minimality of this algorithm.

Theorem 3: Consider a binary arithmetic in precision $p \geq 2$ and the set of all the RN-addition algorithms enhanced with minNum, maxNum, minNumMag and maxNumMag. Among all such algorithms computing the same results $s$ and $t$ as 2Sum on any inputs,

1) each one requires at least 5 operations;

2) each one with 5 operations reduces to Mag2Sum;

3) each one has depth at least 3 .

The proof of Theorems 2 and 3 is much too long to fit here. In summary, the theorems were proved for precisions from 2 to 12 by an exhaustive test on well-chosen inputs, using the GNU MPFR library [10]. The particular form of these inputs (an integer plus a much smaller term) allowed us to generalize these results for any precision larger than 12. The reader can find the full proof in an expanded version of this paper, at http://hal.inria.fr/inria-00475279 (the programs written for the tests are also available).

\section{On the USE OF FAST2SUM IN RADIX-10 ARITHMETIC}

As explained in Section I-B, the Fast2Sum algorithm (Algorithm 1) is proven only when the radix is less than 
or equal to 3 . Indeed, there are counterexamples in radix 10 for this algorithm (we gave one in the introduction). And yet, we are going to show that using Fast2Sum may be of interest, since the very few cases for which it does not return the right answer can easily be enumerated.

\section{A. An analysis of Fast2Sum in radix 10}

In this section, we consider a radix-10 floating-point system of precision $p$. We also consider two floating-point numbers $a$ and $b$, and we will assume $|a| \geq|b|$. Without loss of generality, we assume $a>0$ (just for the sake of simplifying the proofs). Our way of analyzing Fast2Sum will mainly consist in considering Dekker's proof (for radix 2 ) and locating where it does not generalize to decimal arithmetic.

Notice that when the result of a floating-point addition or subtraction is a subnormal, that operation is performed exactly. Due to this, in the following, we assume no underflow in the operations of the algorithm (in case of an underflow, our previous observation implies that the algorithm is correct).

1) First operation: $s \leftarrow R N(a+b)$ : Assume that $a=$ $M_{a} \cdot 10^{e_{a}-p+1}, b=M_{b} \cdot 10^{e_{b}-p+1}$, and $s=M_{s} \cdot 10^{e_{s}-p+1}$, where $M_{a}, M_{b}, M_{s}, e_{a}, e_{b}$, and $e_{s}$ are integers, with

$$
10^{p-1} \leq M_{a},\left|M_{b}\right|,\left|M_{s}\right| \leq 10^{p}-1
$$

Notice that the IEEE 754-2008 standard for floating-point arithmetic does not define the significand and exponent of a decimal number in a unique way. However, in this paper, we will use, without any loss of generality, the values $M_{a}, M_{b}, M_{s}, e_{a}, e_{b}$, and $e_{s}$ that satisfy the above given boundings: what is important is the set of values, not the representations. Define $\delta=e_{a}-e_{b}$.

Since we obviously have $0 \leq a+b \leq 2 a<10 a$, $e_{s}$ is necessarily less than or equal to $e_{a}+1$. We now consider two cases.

- First case: $e_{s}=e_{a}+1$, in that case,

$$
M_{s}=\left\lceil\frac{M_{a}}{10}+\frac{M_{b}}{10^{\delta+1}}\right\rfloor
$$

where $\lceil u\rfloor$ is the integer nearest $u$ (with any of the two possible choices in case of a tie, so that our result applies for the default roundTiesToEven rounding attribute, as well as for the roundTiesToAway attribute defined by IEEE 754-2008). Define $\mu=10 M_{s}-M_{a}$ (notice that $\mu$ is an integer), from

$$
\frac{M_{a}}{10}+\frac{M_{b}}{10^{\delta+1}}-\frac{1}{2} \leq M_{s} \leq \frac{M_{a}}{10}+\frac{M_{b}}{10^{\delta+1}}+\frac{1}{2},
$$

we easily deduce

$$
\frac{M_{b}}{10^{\delta}}-5 \leq \mu \leq \frac{M_{b}}{10^{\delta}}+5,
$$

which implies

$$
|\mu| \leq\left|\frac{M_{b}}{10^{\delta}}\right|+5
$$

From this we conclude that either $s-a$ is exactly representable (with exponent $e_{a}$ and significand $\mu$ ), or we are in the case

$$
\begin{cases}\left|M_{b}\right| \in\left\{10^{p}-4,10^{p}-3,10^{p}-2,10^{p}-1\right\} \\ \text { and } & \delta=0 .\end{cases}
$$

Notice that if $s-a$ is exactly representable, then it will be computed exactly by the second operation.

- Second case: $e_{s} \leq e_{a}$. We have

$$
a+b=\left(10^{\delta} M_{a}+M_{b}\right) \cdot 10^{e_{b}-p+1} .
$$

If $e_{s} \leq e_{b}$ then $s=a+b$ exactly, since $s$ is obtained by rounding $a+b$ to the nearest multiple of $10^{e_{s}-p+1}$, which divides $10^{e_{b}-p+1}$. Hence, $s-a=b$, and $s-a$ is exactly representable.

If $e_{s}>e_{b}$, define $\delta_{2}=e_{s}-e_{b}$. We have

$$
s=\left\lceil 10^{\delta-\delta_{2}} M_{a}+10^{-\delta_{2}} M_{b}\right\rfloor \cdot 10^{e_{s}-p+1},
$$

so that

$$
\begin{array}{r}
\left(10^{-\delta_{2}} M_{b}-\frac{1}{2}\right) \cdot 10^{e_{s}-p+1} \leq s-a \\
\leq\left(10^{-\delta_{2}} M_{b}+\frac{1}{2}\right) \cdot 10^{e_{s}-p+1}
\end{array}
$$

which implies

$$
|s-a| \leq\left(10^{-\delta_{2}}\left|M_{b}\right|+\frac{1}{2}\right) \cdot 10^{e_{s}-p+1} .
$$

Moreover, $e_{s} \leq e_{a} \Rightarrow s-a$ is a multiple of $10^{e_{s}-p+1}$, say $s-a=K \cdot 10^{e_{s}-p+1}$. We get

$$
|K| \leq 10^{-\delta_{2}}\left|M_{b}\right|+\frac{1}{2} \leq \frac{10^{p}-1}{10}+\frac{1}{2} \leq 10^{p}-1,
$$

therefore $s-a$ is exactly representable.

We therefore deduce the following property on the value $s$ computed after the first step.

Property 1: The value $s$ computed by the first operation of Algorithm 1 satisfies:

- either $s-a$ is exactly representable,

- or we simultaneously have

$$
\left\{\begin{array}{l}
\left|M_{b}\right| \in\left\{10^{p}-4,10^{p}-3,10^{p}-2,10^{p}-1\right\}, \\
e_{b}=e_{a} \\
e_{s}=e_{a}+1 .
\end{array}\right.
$$

2) Second and third operations: $z \leftarrow R N(s-a)$ and $t \leftarrow$ $R N(b-z)$ : The second operation is more easily handled. It suffices to notice that when $s-a$ is exactly representable, then $z=s-a$ exactly, so that

$$
b-z=b-(s-a)=(a+b)-s .
$$

This means that when $s-a$ is exactly representable, $b-z$ is the error of the floating-point operation $s \leftarrow$ $R N(a+b)$. Since that error is exactly representable (see for instance [6]), it is computed exactly, so that $t=(a+b)-s$.

Therefore, we deduce the following result.

Theorem 4: If $a$ and $b$ are radix-10 floating-point numbers of precision $p$, with $|a| \geq|b|$, then the value $s$ computed by the first operation of Algorithm 1 satisfies: 
- either $t$ is the error of the floating-point addition $a+b$, which means that $s+t=a+b$ exactly,

- or we simultaneously have

$$
\left\{\begin{array}{l}
\left|M_{b}\right| \in\left\{10^{p}-4,10^{p}-3,10^{p}-2,10^{p}-1\right\} \\
e_{b}=e_{a} \\
e_{s}=e_{a}+1
\end{array}\right.
$$

\section{B. Some consequences}

Let us analyze the few cases for which Algorithm 1 may not work. Notice that since $a$ and $b$ have the same exponent, $|a| \geq|b|$ implies $\left|M_{a}\right| \geq\left|M_{b}\right|$. Also, $\left|M_{a}\right| \leq 10^{p}-1$. Hence, when $\left|M_{b}\right| \in\left\{10^{p}-4,10^{p}-3,10^{p}-2,10^{p}-1\right\}$, the possible values of $\left|M_{a}\right|$ are limited to

- 4 cases for $\left|M_{b}\right|=10^{p}-4$;

- 3 cases for $\left|M_{b}\right|=10^{p}-3$;

- 2 cases for $\left|M_{b}\right|=10^{p}-2$;

- 1 case for $\left|M_{b}\right|=10^{p}-1$.

Also, in these cases, when $a$ and $b$ do not have the same sign, Algorithm 1 obviously works (by Sterbenz Lemma, $s=a+b$ exactly, so that $z=b$ and $t=0)$. Therefore, we can assume that $a$ and $b$ have the same sign. Without loss of generality we assume they are positive. It now suffices to check Algorithm 1 with the 10 possible cases. The results are listed in Table I.

\begin{tabular}{|c|c|c|c|c|}
\hline$M_{a}$ & $\begin{array}{c}M_{b}= \\
10^{p}-4\end{array}$ & $\begin{array}{c}M_{b}= \\
10^{p}-3\end{array}$ & $\begin{array}{c}M_{b}= \\
10^{p}-2\end{array}$ & $\begin{array}{c}M_{b}= \\
10^{p}-1\end{array}$ \\
\hline $10^{p}-4$ & OK & $\mathrm{N} / \mathrm{A}$ & $\mathrm{N} / \mathrm{A}$ & $\mathrm{N} / \mathrm{A}$ \\
\hline $10^{p}-3$ & OK & OK & $\mathrm{N} / \mathrm{A}$ & $\mathrm{N} / \mathrm{A}$ \\
\hline $10^{p}-2$ & OK & $\begin{array}{l}\text { Wrong: } \\
\begin{array}{l}t=-3, \\
(a+b)-s \\
=-5\end{array}\end{array}$ & $\begin{array}{l}\text { Wrong: } \\
t=-2, \\
\begin{array}{l}(a+b)-s \\
=-4\end{array}\end{array}$ & $\mathrm{~N} / \mathrm{A}$ \\
\hline $10^{p}-1$ & $\begin{array}{l}\text { Wrong: } \\
\begin{array}{l}t=-4, \\
(a+b)-s \\
=-5\end{array}\end{array}$ & $\begin{array}{l}\text { Wrong: } \\
t=-3, \\
\begin{array}{c}(a+b)-s \\
=-4\end{array}\end{array}$ & $\begin{array}{l}\text { Wrong: } \\
t=-2, \\
\begin{array}{c}(a+b)-s \\
=-3\end{array}\end{array}$ & $\begin{array}{l}\text { Wrong: } \\
\begin{array}{c}t=-1, \\
(a+b)-s \\
=-2\end{array}\end{array}$ \\
\hline
\end{tabular}

TABLE I

Algorithm 1 IS CHECKED IN THE CASES

$M_{b} \in\left\{10^{p}-4,10^{p}-3,10^{p}-2,10^{p}-1\right\}$ AND $M_{b} \leq M_{a} \leq 10^{p}-1$.

From these results, we notice that there are only 6 cases where Algorithm 1 does not work. This leads us to the following result.

Theorem 5: If $a$ and $b$ are radix-10 floating-point numbers of precision $p$, with $|a| \geq|b|$, then Algorithm 1 always works (i.e., we always have $s+t=a+b$, with $s=R N(a+b))$, unless $a$ and $b$ have the same sign, the same exponent, and their significands $M_{a}$ and $M_{b}$ satisfy:

- $\left|M_{a}\right|=10^{p}-1$ and $\left|M_{b}\right| \geq 10^{p}-4$;

- or $\left|M_{a}\right|=10^{p}-2$ and $\left|M_{b}\right| \geq 10^{p}-3$.

Notice that even in the few (6) cases where Algorithm 1 provides a wrong result, the value of $t$ it returns remains an interesting "correcting term" that can be useful in summation algorithms, since $s+t$ is always closer to $a+b$ than $s$.

Theorem 5 shows that Algorithm Fast2Sum can safely be used in several cases. An example is addition of a constant: for instance, computations of the form " $a \pm 1$ ", quite frequent, can safely be performed whenever $|a| \geq 1$.

Another very frequent case is when one of the operands is known to be significantly larger than the other one (e.g., we add a small correcting term to some estimate).

\section{Halving AND COMPUting The AVERAGE OF TWO NUMBERS IN RADIX 10}

In radix 2 floating-point arithmetic, if $s$ is a floatingpoint number, then $s / 2$ is computed exactly, provided that no underflow occurs. This is not always the case in radix 10 .

Consider a radix-10 number $s$ :

$$
s= \pm S \cdot 10^{e-p+1},
$$

where $S$ is an integer, $10^{p-1} \leq S \leq 10^{p}-1$, and consider the following two cases:

- if $S<2 \cdot 10^{p-1}$, then $5 S$ is less than $10^{p}$, hence $s / 2$ is exactly representable as $5 S \cdot 10^{e-p}$ : it will be computed exactly, with any rounding mode;

- if $S \geq 2 \cdot 10^{p-1}$, then if $S$ is even, $s / 2$ is obviously exactly representable as $S / 2 \cdot 10^{e-p+1}$. If $S$ is odd, let $k$ be the integer such that $S=2 k+1$. From

$$
\frac{s}{2}=\left(k+\frac{1}{2}\right) \cdot 10^{e-p+1},
$$

we deduce that $s / 2$ is a rounding breakpoint for the round-to-nearest mode. Therefore (assuming round to nearest even), the computed value $R N(s / 2)$ will be $k \cdot 10^{e-p+1}$ if $k$ is even, and $(k+1) \cdot 10^{e-p+1}$ otherwise. Let $t$ be that computed result. Notice that $v=2 t$ is either $2 k \cdot 10^{e-p+1}$ or $(2 k+2) \cdot 10^{e-p+1}$ : in any case it is exactly representable, hence it is computed exactly. The same holds for $\delta=s-v$, which will be $\pm 10^{e-p+1}$. This last result is straightforwardly exactly divisible by 2 . We therefore deduce that the sequence of computations $t=R N(0.5 \times s), v=R N(2 \times t)$; $\delta=R N(s-v)$, and $r=R N(0.5 \times \delta)$ will return a value $r$ equal to the error of the floating-point division of $s$ by two (notice that 0.5 is exactly representable in decimal arithmetic).

Now, we easily notice that in all the other cases (that is, when $t$ is exactly $s / 2$ ), the same sequence of operations will return a zero.

This gives us a new error-free transform:

Algorithm 4 (Half-and-error, for radix-10 arithmetic):

$$
\begin{aligned}
t & =R N(0.5 \times s) ; \\
v & =R N(2 \times t) ; \\
\delta & =R N(s-v) ; \\
r & =R N(0.5 \times \delta) ;
\end{aligned}
$$

The following theorem summarizes what we have discussed:

Theorem 6: In radix-10 arithmetic, provided that no underflow occurs (and that $|s|$ is not the largest finite floating-point number), Algorithm 4 returns two values $t$ and $r$ such that $t=R N(s / 2)$, and $t+r=s / 2$ exactly. Also, $r$ is always either 0 or $\pm \frac{1}{2} u l p(s / 2)$. 
Now, let us focus on the computation of the average value of two floating-point numbers $a$ and $b$, namely,

$$
\mu=\frac{a+b}{2} \text {. }
$$

Again, in radix-2 arithmetic, the "naive" method that consists in computing $s=R N(a+b)$ and $m=R N(s / 2)$ (or rather, equivalently, $m=R N(0.5 \times s)$ ) will obviously give $m=R N(\mu)$, unless the addition overflows or the division by 2 underflows. This is not the case in radix 10 . Consider a toy decimal system of precision $p=3$, and the two input numbers $a=1.09$ and $b=0.195$. We get $s=R N(a+b)=1.28$, so that $m=R N(s / 2)=0.640$, whereas the exact average value $\mu$ is 0.6425 : we have an error of 2.5 units in the last place (one can easily show that this is the largest possible error, in the absence of over/underflow).

If a larger precision is available for performing the internal calculations, then we get a better result: if now $s$ is $a+b$ rounded to the nearest in precision $p+d$, then the average value is computed with an error bounded by

$$
\left(\frac{1}{2}+\frac{5}{2} \cdot 10^{-d}\right)
$$

units in the last place.

If no larger precision is available, we may need to use different algorithms. Consider for instance,

Algorithm 5: (Average value in any even radix, when $a$ and $b$ are close)

$$
\begin{aligned}
& d=R N(a-b) ; \\
& h=R N(0.5 \times d) ; \\
& m=R N(a-h) ;
\end{aligned}
$$

Theorem 7: If $a$ and $b$ are two decimal floating-point numbers of the same sign such that $0 \leq b \leq a \leq 2 b$ or $2 b \leq a \leq b \leq 0$, then the value $m$ returned by Algorithm 5 satisfies $m=R N((a+b) / 2)$.

Proof: Without loss of generality we assume that $0 \leq$ $b \leq a \leq 2 b$. Also assume that $a$ and $b$ have been scaled to integers without common factors of 10 , where $b$ has at most $p$ digits. By Sterbenz Lemma we have $R N(a-b)=a-b$. The proof is now split in two cases:

$\boldsymbol{a}-\boldsymbol{b}$ is even: $(a-b) / 2$ is exactly representable. Hence $m=R N(a-R N((a-b) / 2))=R N((a+b) / 2)$.

$\boldsymbol{a}-\boldsymbol{b}$ is odd: $R N((a-b) / 2)=(a-b) / 2+\delta(\delta= \pm 1 / 2)$ is exactly computable and representable as a $p$-digit even integer (since mid-points round to even). Now assume that $a$ is a $p+k$ digit integer, with $k$ minimal. Then it follows that $k \leq 1$. Consider:

$$
R N((a-b) / 2)=(a-b) / 2+\delta,
$$

from which it follows that

$$
a-R N((a-b) / 2)=(a+b) / 2-\delta(=(a+b \pm 1) / 2),
$$

which is an integer representable on at most $p+k$ digits (since $a$ and $R N((a-b) / 2)$ have the same sign and both are integers representable on $p+k$ digits).

If $k=0$, then obviously $m=R N(a-R N((a-b) / 2))=$ $R N((a+b) / 2)$.
If $k=1$, then $a$ is even, hence $a-R N((a-b) / 2)$ is even, thus not a mid point. Hence rounding to $p$ digits yields $m=R N(a-R N((a-b) / 2))=R N((a+b) / 2)$.

\section{ON The IMPOSSIBILITY OF COMPUTING A ROUND-TO-NEAREST SUM}

In this section, we are interested in the computation of the sum of $n$ floating-point numbers, correctly rounded to nearest. We prove the following result.

Theorem 8: Let $a_{1}, a_{2}, \ldots, a_{n}$ be $n \geq 3$ floating-point numbers of the same format. Assuming an unbounded exponent range, and assuming that the radix of the floatingpoint system is even, an $\mathrm{RN}$-addition algorithm cannot always return $R N\left(a_{1}+a_{2}+\cdots+a_{n}\right)$.

If there exists an $\mathrm{RN}$-addition algorithm to compute the round-to-nearest sum of $n$ floating-point numbers, with $n \geq 3$, then this algorithm must also compute the roundto-nearest sum of 3 floating-point values. As a consequence we only consider the case $n=3$ in the proof of this theorem. We show how to construct for any $\mathrm{RN}$-algorithm a set of input data such that the result computed by the algorithm differs from the round-to-nearest result.

Proof of Theorem 8: Assume a radix- $\beta$ arithmetic, where $\beta$ is even. An RN-addition algorithm can be represented by a directed acyclic graph ${ }^{4}$ (DAG) whose nodes are the arithmetic operations. Given such an algorithm, let $r$ be the depth of its associated graph. First we consider the input values $a_{1}, a_{2}, a_{3}$ defined as follows.

- For a given ${ }^{5}$ integer $k$, we choose $a_{1}=\beta^{k+p}$ and $a_{2}=\left(\frac{\beta}{2}\right) \beta^{k}: a_{1}$ and $a_{2}$ are two nonzero multiples of $\beta^{k}$ whose sum is the exact middle of two consecutive floating-point numbers;

- $a_{3}=\varepsilon$, with $0 \leq \beta^{r-1}|\varepsilon| \leq \beta^{k-p-1}$ for $r \geq 1$.

Note that when $\varepsilon \neq 0$,

$$
R N\left(a_{1}+a_{2}+a_{3}\right)=\left\{\begin{array}{lll}
R D\left(a_{1}+a_{2}+a_{3}\right) & \text { if } & \varepsilon<0 \\
R U\left(a_{1}+a_{2}+a_{3}\right) & \text { if } & \varepsilon>0,
\end{array}\right.
$$

where we may also conclude that $R N\left(a_{1}+a_{2}+a_{3}\right) \geq \beta^{k+p}$.

The various computations that can be performed "at depth 1", i.e., immediately from the input to the algorithm are illustrated below. The value of $\varepsilon$ is so small that after rounding to nearest, every operation with $\varepsilon$ in one of its entries will return the same value as if $\varepsilon$ were zero, unless the other entry is 0 or $\varepsilon$.
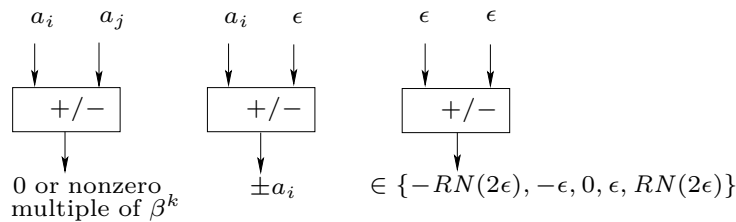

An immediate consequence is that after these computations "at depth 1", the possible available variables

\footnotetext{
${ }^{4}$ Such an algorithm cannot have "while" loops, since tests are prohibited. It may have "for" loops that can be unrolled.

${ }^{5}$ Here $k$ is arbitrary. When considering a limited exponent range, we have to assume that $k+p$ is less than the maximum exponent.
} 
are nonzero multiples of $\beta^{k}$ that are the same as if $\varepsilon$ were 0 , and values bounded by $R N(2 \varepsilon)$, thus by $\beta|\varepsilon|$ in absolute value. By induction one easily shows that the available variables after a computation of depth $m$ are either nonzero multiples of $\beta^{k}$ that are the same as if $\varepsilon$ were 0 , or values bounded by $\beta^{m}|\varepsilon|$ in absolute value.

Now, consider the very last addition/subtraction, at depth $r$ in the DAG of the RN-addition algorithm. Since $R N\left(a_{1}+a_{2}+a_{3}\right) \geq \beta^{k+p}$, one of the inputs of this last operation is a nonzero multiple of $\beta^{k}$ that is the same as if $\varepsilon$ were 0 , and the other input is either also a nonzero multiple of $\beta^{k}$ or a value bounded by $\beta^{r-1}|\varepsilon|$ in absolute value. In both cases the result does not depend on the sign of $\varepsilon$, hence it is always possible to choose the sign of $\varepsilon$ so that the round-to-nearest result differs from the computed one.

In the proof of Theorem 8, it was necessary to assume an unbounded exponent range to make sure that with a computational graph of depth $r$, we can always build an $\varepsilon$ so small that $\beta^{r-1} \varepsilon$ vanishes when added to any nonzero multiple of $\beta^{k}$. This constraint can be transformed into a constraint on $r$ related to the extremal exponents $e_{\min }$ and $e_{\max }$ of the floating-point system. For instance, in radix 2 , assuming $\varepsilon= \pm 2^{e_{\min }}$ and $a_{1}=2^{k+p}=2^{e_{\max }}$, the inequality $2^{r-1}|\varepsilon| \leq 2^{k-p-1}$ gives the following theorem.

Theorem 9: Let $a_{1}, a_{2}, \ldots, a_{n}$ be $n \geq 3$ floating-point numbers of the same binary format. Assuming the extremal exponents of the floating-point format are $e_{\min }$ and $e_{\max }$, an RN-addition algorithm of depth $r$ cannot always return $R N\left(a_{1}+a_{2}+\cdots+a_{n}\right)$ as soon as

$$
r \leq e_{\max }-e_{\min }-2 p \text {. }
$$

For instance, with the IEEE 754-1985 double precision format $\left(e_{\min }=-1022, e_{\max }=1023, p=53\right)$, an RNaddition algorithm able to always evaluate the round-tonearest sum of at least 3 floating-point numbers (if such an algorithm exists!) must have depth at least 1939.

\section{Correctly-Rounded Sums of THREE FLOATING-POINT NUMBERS}

We have proved in the previous section that there exist no RN-addition algorithms of acceptable size to compute the round-to-nearest sum of $n \geq 3$ floating-point values. In [5], Boldo and Melquiond presented an algorithm to compute $R N(a+b+c)$ using a "round-to-odd" addition. Rounding to odd is defined as follows:

Definition 1 (Rounding to odd):

- if $x$ is a floating-point number, then $R O(x)=x$;

- otherwise, $R O(x)$ is the value among $R D(x)$ and $R U(x)$ whose least significant digit is odd.

The algorithm of Boldo and Melquiond for computing of $R N(a+b+c)$ is depicted on Fig. 1. Boldo and Melquiond proved their algorithm (provided no overflow occurs) in radix 2 , yet it can be checked that it also works in radix 10 .

Rounding to odd is not a rounding mode available on current architectures, hence a software emulation was proposed in [5] for radix 2: this software emulation requires accesses to the binary representation of the floating-point numbers and conditional branches, both of which are costly on pipelined architectures.

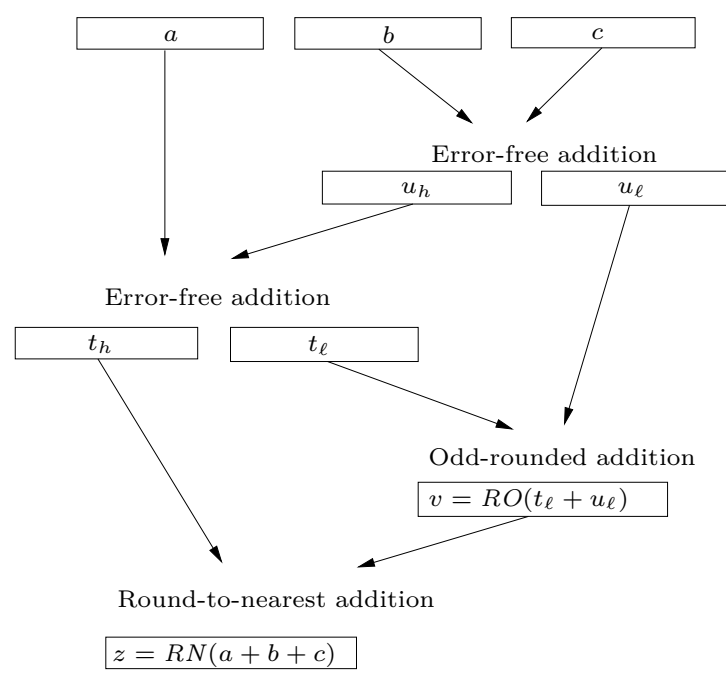

Fig. 1. The Boldo-Melquiond algorithm.

In the next section, we propose a new algorithm for simulating the round-to-odd addition of two floatingpoint values. This algorithm uses only available IEEE-754 rounding modes and a multiplication by the constant 0.5 (and only in a case where this multiplication is exact), and can be used to avoid access to the binary representation of the floating-point numbers and conditional branches in the computation of $R N(a+b+c)$ with the Boldo-Melquiond algorithm. We also study a modified version of the BoldoMelquiond algorithm to compute $D R(a+b+c)$, where $D R$ denotes any of the IEEE-754 directed rounding modes.

\section{A. A new method for rounding to odd}

If we allow multiplication by the constant 0.5 and choosing the rounding mode for each operation, the following algorithm can be used to implement the round-to-odd addition, assuming that the radix $\beta$ of the floating-point system is even.

For some of the arithmetic operations performed in this algorithm, the result is exactly representable, so it will be exactly computed with any rounding mode: hence, for these operations, we have not indicated a particular rounding mode.

Algorithm 6 (OddRoundSum(a,b), arbitrary even radix):

$$
\begin{array}{llll}
d & =R D(a+b) ; & \\
u & =R U(a+b) ; & \\
u l p & =u-d ; & & \text { exact\} } \\
h u l p & =0.5 \times u l p ; & & \text { exact\} } \\
e & =R N(d+h u l p) ; & \\
o^{\prime} & =u-e ; & \text { exact\} } \\
o & =o^{\prime}+d ; & \text { exact }
\end{array}
$$


For instance, with $\beta=10, p=4, a=2.355$, and $b=$ 0.8935 , we successively get $d=3.248, u=3.249$, ulp $=$ 0.001 , hulp $=0.0005, e=3.248, o^{\prime}=0.001$, and $o=3.249$.

Theorem 10: Let $a$ and $b$ be two floating-point numbers, and assume that $a+b$ does not overflow and that " $R N$ " means round to nearest even. Then Algorithm 6 computes $o=R O(a+b)$.

Proof: Since the radix is even, $0.5=1 / 2$ is exactly representable. If $a+b$ is exactly representable, then all the operations are exact and $d=u=a+b, h u l p=u l p=0$, $e=d, o^{\prime}=0$, and $o=d=a+b$.

Otherwise $d$ and $u$ are consecutive machine numbers and $u l p$ is a power of the (even) radix, which cannot be the minimum nonzero machine number in magnitude (because an exact representation of $a+b$ takes at least $p+1$ digits). Thus $u l p / 2$ is exactly representable, so that $d+h u l p$ is the exact middle of $d$ and $u$. Therefore, by the round-tonearest-even rule, $e$ is the value, among $d$ and $u$, whose last significand digit is even. Then $o$ is the other one, which is the desired result.

The only case when this algorithm does not return the correctly rounded-to-odd value is the (extremely rare) case when $R U(a+b)$ is infinite whereas $R D(a+b)$ is not.

When the radix is 2 , it is possible to save an operation, by replacing the three instructions $u l p=u-d$, hulp $=$ $0.5 \times u l p$, and $e=R N(d+h u l p)$ of Algorithm 6 by the two instructions $e^{\prime}=R N(d+u)$ and $e=e^{\prime} \times 0.5$. Note that if $e^{\prime} \times 0.5$ is in the subnormal range, this means that $a+b$ is also in the subnormal range, implying that $d=u$, and $e^{\prime} \times 0.5$ is performed exactly.

Algorithm 6 or its binary variant can be used in the algorithm depicted on Fig. 1 to implement the round-toodd addition. Then we obtain an algorithm using only basic floating-point operations and the IEEE-754 rounding modes to compute $R N(a+b+c)$ for all floating-point numbers $a, b$ and $c$.

In Algorithm 6 and its binary variant, note that $d$ and $u$ may be calculated in parallel and that the calculation of hulp and $e$ (in the general case, i.e., Algorithm 6 ) or $e$ and $o^{\prime}$ (in the binary case) may be combined if a fused multiply-add (FMA) instruction is available. On most floating-point units, the rounding mode is dynamic and changing it requires flushing the pipeline, which is expensive. However, on some processors such as Intel's Itanium, the rounding mode of each floating-point operation can be chosen individually [7, Chap. 3]. In this case, the choice of rounding mode has no impact on the running time of a sequence of floating-point operations. Moreover the Itanium provides an FMA instruction, hence the proposed algorithm can be expected to be a very efficient alternative to compute round-to-odd additions on this processor.

\section{B. Computation of $\operatorname{DR}(a+b+c)$}

We now focus on the problem of computing $D R(a+b+c)$, where $D R$ denotes one of the directed rounding modes ( $R Z, R D$ or $R U$ ). The algorithm we consider for $D R=R D$ or $R U$ (the case $D R=R Z$ will be dealt with later) is a variant of the Boldo-Melquiond algorithm. The only difference is that the last two operations use a directed rounding mode. The algorithm can be summarized as follows.

$$
\begin{aligned}
\text { Algorithm 7 }(D R 3(a, b, c)) & \text { : } \\
\left(u_{h}, u_{\ell}\right) & =2 \operatorname{Sum}(b, c) ; \\
\left(t_{h}, t_{\ell}\right) & =2 \operatorname{Sum}\left(a, u_{h}\right) ; \\
v & =D R\left(t_{\ell}+u_{\ell}\right) ; \\
z & =D R\left(t_{h}+v\right) ;
\end{aligned}
$$

Algorithm 7 computes $\operatorname{DR}(a+b+c)$ for rounding downward or upward. However, it may give an incorrect answer for rounding toward zero.

To prove Algorithm 7, we need to distinguish between different precisions. To that purpose, we introduce some notation. Let $\mathcal{F}_{\beta, p}$ denote the set of all radix- $\beta$, precision$p$ floating-point numbers, with an unbounded exponent range (where, obviously, $\beta \geq 2$ and $p \geq 1$ ). Given $x \in \mathbb{R}$, we shall denote $x$ rounded downward, rounded upward, rounded toward zero and rounded to nearest in $\mathcal{F}_{\beta, p}$ by $R D_{p}(x), R U_{p}(x), R Z_{p}(x)$ and $R N_{p}(x)$ respectively. Note that even though these functions depend on the parameter $\beta$, we omit $\beta$ from their indices to make the notation simpler, since $\beta$ is regarded as fixed; we will even omit the index $p$ when only precision $p$ is considered, just like in the other sections of the paper.

Theorem 11: If the radix $\beta$ and the precision $p$ satisfy

- either $5 \cdot \beta^{1-p} \leq 1$,

- or $\beta=2^{k}$, where $k \geq 1$ is an integer, and $3 \cdot \beta^{1-p} \leq 1$. Then, given $a, b, c \in \mathcal{F}_{\beta, p}$, and $s=a+b+c$ the exact sum, and provided no overflow occurs, algorithm DR3 (Algorithm 7) computes $z=D R(s)$.

Notice that the conditions of Theorem 11 become $p \geq 3$ in radix 2 , and $p \geq 2$ in radix 10 .

The assumption that no overflow occurs cannot be suppressed: for instance, if $b+c$ overflows whereas the sum $a+b+c$ is smaller than the overflow threshold, the algorithm does not work. Underflow is easily dealt with: it does not hinder the result.

For proving Theorem 11, we use the next two lemmata.

Lemma 12: Let $\beta \geq 2$ and two precisions $p$ and $q$ such that $q \geq p$. Let $D R$ be one of the directed rounding modes $(R Z, R D$ or $R U)$, so that $D R_{p}$ and $D R_{q}$ denote the corresponding rounding functions in $\mathcal{F}_{\beta, p}$ and $\mathcal{F}_{\beta, q}$ respectively. Then for all $x \in \mathbb{R}, D R_{p}(x)=D R_{p}\left(D R_{q}(x)\right)$.

The proof of Lemma 12 mainly relies on $\mathcal{F}_{\beta, p} \subset \mathcal{F}_{\beta, q}$ and on the fact that both roundings are done in the same direction.

Lemma 13: Let $\beta \geq 2, p \geq 1$, and $x, y \in \mathcal{F}_{\beta, p}$ such that $x+y \notin \mathcal{F}_{\beta, p}$. We denote $z=R N(x+y)$.

- If $\beta=2^{k}$, where $k \geq 1$ is an integer, then $|y| \leq 2|z|$.

- For any radix $\beta,|y| \leq 2\left(1+\beta^{1-p}\right)|z|$.

Proof: First, since $x+y \notin \mathcal{F}_{\beta, p}$, neither $x$ nor $y$ can be 0 . If $x$ and $y$ have the same sign, then $|y| \leq|z| \leq 2|z|$. In the following, let us assume that $x$ and $y$ have different signs. Under this condition, Sterbenz's lemma yields: If $\frac{1}{2}|y| \leq|x| \leq 2|y|$, then $x+y \in \mathcal{F}_{\beta, p}$. Since by assumption $x+y \notin \mathcal{F}_{\beta, p}$, 
- either $\frac{1}{2}|y|>|x|$, hence $|x+y|=|y|-|x|>\frac{1}{2}|y|$,

- or $|x|>2|y|$, hence $|x+y|=|x|-|y|>|y|$.

In both cases, $|x+y| \geq \frac{1}{2}|y|$, hence $|z|=R N(|x+y|) \geq$ $R N\left(\frac{1}{2}|y|\right)$. If $\beta$ is a power of two, then $R N\left(\frac{1}{2}|y|\right)=\frac{1}{2}|y|$, hence $|z| \geq \frac{1}{2}|y|$. If no assumption is made on the radix $\beta$, then we write $R N\left(\frac{1}{2}|y|\right)=(1+\varepsilon) \frac{1}{2}|y|$, with $|\varepsilon| \leq \frac{1}{2} \beta^{1-p}$, which implies $R N\left(\frac{1}{2}|y|\right) \geq \frac{1}{2}\left(1-\frac{1}{2} \beta^{1-p}\right)|y|$. A quick calculation shows that

$$
\frac{1}{1-\frac{1}{2} \beta^{1-p}} \leq\left(1+\beta^{1-p}\right)
$$

as a consequence, $|y| \leq 2\left(1+\beta^{1-p}\right)|z|$.

Proof of Theorem 11: In this proof, let us denote $t_{\ell}+u_{\ell}$ by $\gamma$, and $t_{h}+v$ by $s^{\prime}$.

The following two special cases are easily handled:

- If $a+u_{h} \in \mathcal{F}_{\beta, p}$, then $t_{\ell}=0$, which means that $s=$ $t_{h}+u_{\ell} ;$ moreover, $z=D R\left(t_{h}+v\right)=D R\left(t_{h}+u_{\ell}\right)$, hence $z=D R(s)$.

- If $\gamma=0$, then $s=t_{h}, v=0$, and $z=D R(s)$.

Let us now assume that $a+u_{h} \notin \mathcal{F}_{\beta, p}$ and $\gamma \neq 0$. Since $\left(t_{h}, t_{\ell}\right)=2 \operatorname{Sum}\left(a, u_{h}\right)$, then $\left|t_{\ell}\right| \leq \frac{1}{2} \beta^{1-p}\left|t_{h}\right|$, and from $|\gamma| \leq\left|u_{\ell}\right|+\left|t_{\ell}\right|$ we deduce that $|\gamma| \leq\left|u_{\ell}\right|+\frac{1}{2} \beta^{1-p}\left|t_{h}\right|$. On the other hand, since $\left(u_{h}, u_{\ell}\right)=2 \operatorname{Sum}(b, c)$, then $\left|u_{\ell}\right| \leq$ $\frac{1}{2} \beta^{1-p}\left|u_{h}\right|$. As a consequence,

$$
|\gamma| \leq \frac{1}{2} \beta^{1-p}\left|u_{h}\right|+\frac{1}{2} \beta^{1-p}\left|t_{h}\right| .
$$

As $\left(t_{h}, t_{\ell}\right)=2 \operatorname{Sum}\left(a, u_{h}\right)$ and $t_{h}=R N\left(a+u_{h}\right)$, and since $a+u_{h}$ does not belong to $\mathcal{F}_{\beta, p}$ by hypothesis, Lemma 13 can be used to bound $\left|u_{h}\right|$ with respect to $\left|t_{h}\right|$. We distinguish two cases.

- If $\beta$ is a power of two, then $\left|u_{h}\right| \leq 2\left|t_{h}\right|$. As a consequence $|\gamma| \leq \frac{3}{2} \beta^{1-p}\left|t_{h}\right|$, and since $3 \beta^{1-p} \leq 1$, $|\gamma| \leq\left|t_{h}\right|$. From $|s|=\left|t_{h}+t_{\ell}+u_{\ell}\right| \geq\left|t_{h}\right|-|\gamma|$, we also deduce $|s| \geq\left(\frac{2}{3} \beta^{p-1}-1\right)|\gamma|$. Since $3 \beta^{1-p} \leq 1$ implies $\frac{2}{3} \beta^{p-1}-1 \geq 1$, also $|\gamma| \leq|s|$.

- Otherwise, one has $\left|u_{h}\right| \leq 2\left(1+\beta^{1-p}\right)\left|t_{h}\right|$, which gives $|\gamma| \leq\left(\frac{3}{2}+\beta^{1-p}\right) \beta^{1-p}\left|t_{h}\right| \leq \frac{5}{2} \beta^{1-p}\left|t_{h}\right|$, and since $5 \beta^{1-p} \leq 1,|\gamma| \leq\left|t_{h}\right|$ follows. As $|s| \geq\left|t_{h}\right|-|\gamma|$, then $|s| \geq\left(\frac{2}{5} \beta^{p-1}-1\right)|\gamma|$. Since $5 \beta^{1-p} \leq 1$ implies $\frac{2}{5} \beta^{p-1}-1 \geq 1$, it follows that $|\gamma| \leq|s|$.

Therefore, in both cases we have

$$
|\gamma| \leq\left|t_{h}\right| \text { and }|\gamma| \leq|s| .
$$

We now focus on the last two operations in Algorithm 7 . Defining $\rho_{D R}(x)$ by $\rho_{R D}(x)=\lfloor x\rfloor$ and $\rho_{R U}(x)=\lceil x\rceil$, one has

$$
s^{\prime}=t_{h}+D R_{p}\left(t_{\ell}+u_{\ell}\right)=t_{h}+\rho_{D R}\left(\frac{\gamma}{\operatorname{ulp}_{p}(\gamma)}\right) u \operatorname{lp}_{p}(\gamma) .
$$

From the first inequality in (1) it follows that $\operatorname{ulp}_{p}(\gamma) \leq$ $\operatorname{ulp}_{p}\left(t_{h}\right)$, which implies that $t_{h}$ is an integral multiple of $\operatorname{ulp}_{p}(\gamma)$. Since $s=t_{h}+\gamma$, we write

$$
\begin{aligned}
s^{\prime} & =\left(\frac{t_{h}}{\operatorname{ulp}_{p}(\gamma)}+\rho_{D R}\left(\frac{\gamma}{\operatorname{ulp}_{p}(\gamma)}\right)\right) \operatorname{ulp}_{p}(\gamma) \\
& =\rho_{D R}\left(\frac{s}{\operatorname{ulp}_{p}(\gamma)}\right) \operatorname{ulp}_{p}(\gamma) .
\end{aligned}
$$

Since $\gamma \neq 0$ and $s \neq 0$, there exists an integer $q$ such that $\operatorname{ulp}_{p}(\gamma)=\operatorname{ulp}_{q}(s) .{ }^{6}$ Furthermore, it follows from the second inequality in (1) that $\operatorname{ulp}_{p}(\gamma) \leq \operatorname{ulp}_{p}(s)$, hence $\operatorname{ulp}_{q}(s) \leq \operatorname{ulp}_{p}(s)$, which implies $q \geq p$. Hence

$$
s^{\prime}=\rho_{D R}\left(\frac{s}{\operatorname{ulp}_{q}(s)}\right) \operatorname{ulp}_{q}(s)=D R_{q}(s) .
$$

Since $z=D R_{p}\left(s^{\prime}\right)$, one has $z=D R_{p}\left(D R_{q}(s)\right)$. Then from Lemma 12 , we obtain $z=D R_{p}(s)$.

The proof cannot be extended to $R Z$, due to the fact that the two roundings can be done in opposite directions. For instance, if $s>0$ (not exactly representable) and $t_{\ell}+u_{\ell}<0$, then one has $R D(s) \leq R D\left(s^{\prime}\right)$ as wanted, but $t_{\ell}+u_{\ell}$ rounds upward and $s^{\prime}$ can be $R U(s)$, so that $z=R U(s)$ instead of $R Z(s)=R D(s)$, as shown on the following counter-example. In radix 2 and precision 7 , with $a=-3616, b=19200$ and $c=-97$, we have $s=15487$, $R Z(s)=15360$ and $R U(s)=15488$. Running Algorithm 7 on this instance gives $z=15488$, so that $R U(s)$ has been computed instead of $R Z(s)$.

Nevertheless $R Z(s)$ can be obtained by computing both $R D(s)$ and $R U(s)$, then selecting the one closer to zero using the minNumMag instruction [11]. This algorithm for computing $R Z(a+b+c)$ without branches can already be implemented on the Itanium architecture thanks to the famin instruction [7].

\section{Conclusions}

We have proved that in binary arithmetic Knuth's 2Sum algorithm is minimal, both in terms of the number of operations and the depth of the dependency graph. We have investigated the possibility of using the Fast2Sum algorithm in radix-10 floating-point arithmetic. We have also shown that, just by performing round-to-nearest floatingpoint additions and subtractions without any testing, it is impossible to compute the round-to-nearest sum of $n \geq 3$ floating-point numbers in even-radix arithmetic. If changing the rounding mode is allowed, in even-radix arithmetic, we can implement, without testing, the nonstandard rounding to odd defined by Boldo and Melquiond, which makes it indeed possible to compute the sum of three floating-point numbers rounded to nearest. We finally proposed an adaptation of the Boldo-Melquiond algorithm for calculating $a+b+c$ rounded according to the standard directed rounding modes.

\section{ACKNOWLEDGEMENT}

We thank Damien Stehlé, who actively participated in our first discussions on these topics.

\section{REFERENCES}

[1] W. Kahan. Pracniques: further remarks on reducing truncation errors. Commun. ACM, 8(1):40, 1965.

\footnotetext{
${ }^{6}$ Notice that $q$ may be negative. We use the same definition of ulp $_{q}$ as previously: if $\beta^{e} \leq|x|<\beta^{e+1}$ with $e \in \mathbb{Z}$, then $\operatorname{ulp}_{q}(x)=\beta^{e+1-q}$.
} 
[2] American National Standards Institute and Institute of Electrical and Electronic Engineers. IEEE Standard for Binary Floating-Point Arithmetic, ANSI/IEEE Standard 754-1985. New York, 1985.

[3] American National Standards Institute and Institute of Electrical and Electronic Engineers. IEEE Standard for Radix Independent Floating-Point Arithmetic, ANSI/IEEE Standard 854-1987. New York, 1987.

[4] E. Adams and U. Kulisch, editors. Scientific Computing with Automatic Result Verification. Academic Press, San Diego, 1993.

[5] S. Boldo and G. Melquiond. Emulation of a FMA and correctlyrounded sums: proved algorithms using rounding to odd. IEEE Transactions on Computers, 57(4), Apr. 2008.

[6] S. Boldo and M. Daumas. Representable correcting terms for possibly underflowing floating point operations. In J.-C. Bajard and M. Schulte, editors, Proceedings of the 16th Symposium on Computer Arithmetic, pages 79-86. IEEE Computer Society Press, Los Alamitos, CA, 2003.

[7] M. Cornea, J. Harrison, and P. T. P. Tang. Scientific computing on Itanium based systems. Intel Press, 2002.

[8] T. J. Dekker. A floating-point technique for extending the available precision. Numerische Mathematik, 18(3):224-242, 1971.

[9] J. R. Hauser. Handling floating-point exceptions in numeric programs. ACM Trans. Program. Lang. Syst., 18(2):139-174, 1996.

[10] L. Fousse, G. Hanrot, V. Lefèvre, P. Pélissier, and P. Zimmermann. MPFR: A multiple-precision binary floating-point library with correct rounding. ACM Transactions on Mathematical Software, 33(2), 2007. Available at http://www.mpfr.org/.

[11] IEEE Computer Society. IEEE Standard for Floating-Point Arithmetic. IEEE Standard 754-2008, Aug. 2008. Available at http://ieeexplore.ieee.org/servlet/opac?punumber $=4610933$.

[12] P. Kornerup, V. Lefèvre, N. Louvet, and J.-M. Muller. On the Computation of Correctly-Rounded Sums Proceedings of the 19th IEEE Symposium on Computer Arithmetic Portland, OR, June 2009.

[13] D. Knuth. The Art of Computer Programming, 3rd edition, volume 2. Addison-Wesley, Reading, MA, 1998.

[14] O. Møller. Quasi double-precision in floating-point addition. $B I T, 5: 37-50,1965$.

[15] D. Monniaux. The pitfalls of verifying floating-point computations. ACM TOPLAS, 30(3):1-41, 2008. Available at http://hal.archives-ouvertes.fr/hal-00128124.

[16] J.-M. Muller, N. Brisebarre, F. de Dinechin, C.-P. Jeannerod, V. Lefèvre, G. Melquiond, N. Revol, D. Stehlé, and S. Torres. Handbook of Floating-Point Arithmetic. Birkhäuser, 2009.

[17] T. Ogita, S. M. Rump, and S. Oishi. Accurate sum and dot product. SIAM Journal on Scientific Computing, 26(6):1955$1988,2005$.

[18] M. Pichat. Correction d'une somme en arithmétique à virgule flottante (in French). Numerische Mathematik, 19:400-406, 1972.

[19] D. Priest. On Properties of Floating-Point Arithmetics: Numerical Stability and the Cost of Accurate Computations. PhD thesis, University of California at Berkeley, 1992.

[20] S. M. Rump, T. Ogita and S. Oishi. Accurate Floating-Point Summation Part I: Faithful Rounding. SIAM Journal on Scientific Computing, 31(1):189-224, 2008.

[21] J. R. Shewchuk. Adaptive Precision Floating-Point Arithmetic and Fast Robust Geometric Predicates. Discrete 8 Computational Geometry, 18:305-363, 1997.

[22] Y.-K. Zhu, J.-H. Yong, and G.-Q. Zheng. A New Distillation Algorithm for Floating-Point Summation. SIAM Journal on Scientific Computing, 26(6):2066-2078, 2005.

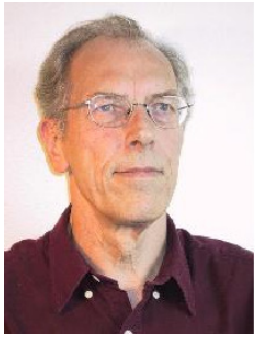

Peter Kornerup received the Mag. Scient. degree in mathematics from Aarhus University, Denmark, in 1967. After a period with the University Computing Center, from 1969 involved in establishing the computer science curriculum at Aarhus University, he helped found the Computer Science Department there in 1971 and served as its chairman until in 1988, when he became Professor of Computer Science at Odense University, now University of Southern Denmark. Prof. Kornerup has served on program committees for numerous IEEE, ACM and other meetings, in particular he has been on the Program Committees for the 4th through the 19th IEEE Symposium on Computer Arithmetic, and served as Program Co-Chair for these symposia in 1983, 1991, 1999 and 2007. He has been guest editor for a number of journal special issues, and served as an associate editor of the IEEE Transactions on Computers from 1991 to 1995 . He is a member of the IEEE Computer Society.

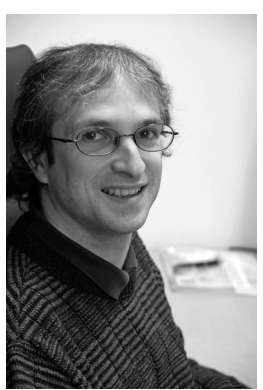

Vincent Lefèvre received the MSc and $\mathrm{PhD}$ degrees in computer science from the École Normale Supérieure de Lyon, France, in 1996 and 2000, respectively. He has been an INRIA researcher at the LORIA, France, from 2000 to 2006, and at the LIP, ENS-Lyon, France, since 2006. His research interests include computer arithmetic. He participated in the revision of the IEEE 754 standard.

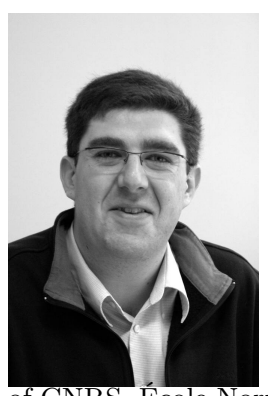

Nicolas Louvet received the MSc degree from the Université de Picardie Jules Verne (Amiens, France), in 2004, and the PhD degree in computer science from the Université de Perpignan Via Domitia (Perpignan, France) in 2007. After being an INRIA postdoctoral fellow in the Arénaire reasearch team, he is now assistant professor in the department of computer science of the Université Claude Bernard Lyon 1 (Lyon, France), and a member of the LIP laboratory (LIP is a joint laboratory of CNRS, École Normale Supérieure de Lyon, INRIA and Université Claude Bernard Lyon 1). His research interests are in computer arithmetic.

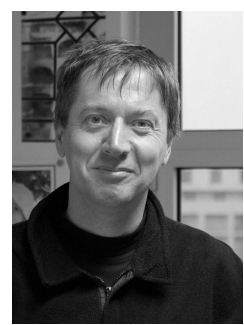

Jean-Michel Muller was born in Grenoble, France, in 1961. He received his Ph.D. degree in 1985 from the Institut National Polytechnique de Grenoble. He is Directeur de Recherches (senior researcher) at CNRS, France, and he is the former head of the LIP laboratory (LIP is a joint laboratory of CNRS, Ecole Normale Supérieure de Lyon, INRIA and Université Claude Bernard Lyon 1). His research interests are in Computer Arithmetic. Dr. Muller was co-program chair of the 13th IEEE Symposium on Computer Arithmetic (Asilomar, USA, June 1997), general chair of SCAN'97 (Lyon, France, sept. 1997), general chair of the 14th IEEE Symposium on Computer Arithmetic (Adelaide, Australia, April 1999). He is the author of several books, including "Elementary Functions, Algorithms and Implementation" (2nd edition, Birkhäuser Boston, 2006), and he coordinated the writing of the "Handbook of Floating-Point Arithmetic (Birkhäuser Boston, 2010). He served as associate editor of the IEEE Transactions on Computers from 1996 to 2000 . He is a senior member of the IEEE. 